\title{
Liquid Chromatography High-resolution Mass Spectrometric QUAL/QUAN Approaches for Drug Metabolism and Metabolomics
}

\author{
David Tonoli§, Emmanuel Varesio, and Gérard Hopfgartner* \\ §SCS-Metrohm Foundation Award for best oral presentation
}

\begin{abstract}
A liquid chromatography-high-resolution mass spectrometry platform was used for simultaneous qualitative and quantitative (QUAL/QUAN) acquisition, enabling drug metabolism and metabolomics investigations. Plasma study samples were monitored for three different groups of patients at a single time-point ( $1 \mathrm{~h}$ after drug administration): one group received acetaminophen (APAP), one group received both APAP and ketorolac and one group was a control group. The quantification of APAP and two of its metabolites (APAP-glucuronide and APAP-cysteine) was performed on a fast acquisition quadrupole-Time-Of-Flight (50-100 ms duty cycle, resolving power of 30,000 ) compatible with UHPLC time constraints. High-resolution Selected Reaction Monitoring was used for quantification of APAP and its metabolites from $50-10,000 \mathrm{ng} / \mathrm{mL}$ using a $50 \mu \mathrm{L}$ plasma aliquot. Average measured concentrations were for APAP $6,650 \mathrm{ng} / \mathrm{mL}$ vs $6,160 \mathrm{ng} / \mathrm{mL}$, APAP-CYS concentrations were 154.2 $\mathrm{ng} / \mathrm{mL}$ vs $140.6 \mathrm{ng} / \mathrm{mL}$ and APAP-GLU concentrations $8,750 \mathrm{ng} / \mathrm{mL}$ vs $8,430 \mathrm{ng} / \mathrm{mL}$ between the group that received only APAP $(n=11)$ and the group that received APAP in combination with ketorolac $(n=11)$. No major differences were observed between the two groups of patients, as it would be expected due to the differing metabolism pathway for both substances. For the qualitative aspect, a metabolomics data processing platform with biological QC samples was applied to the study samples to search for unanticipated metabolites and biomarkers related to APAP and ketorolac metabolism. Multivariate analysis (i.e. Principle Component Analysis), variables grouping tools (i.e. PCVG) and high-resolution MS(MS) spectra from the $\mathrm{MS}^{\mathrm{ALL}}$ acquisition strategy enabled the profiling and characterization of circulating metabolites of APAP in plasma such as APAP-sulfate, APAP-mercapturate as well as ketorolac.
\end{abstract}

Keywords: Acetaminophen · High resolution · Mass spectrometry · Metabolomics · UHPLC

\section{Introduction}

In the course of drug development, one aims to correlate the desired pharmacological activity to the quantitative measurement of plasmatic concentrations of the drug in order to maintain the patient within the therapeutic window. ${ }^{[1]}$ However, diverse mechanisms, mainly related to the pharmacological activity itself and to the metabolism of the parent drug, change the plasmatic concentration over time. Therefore it is important at an early stage to fully characterize the metabolism mechanism participating in the modulation of

${ }^{*}$ Correspondence: Prof. G. Hopfgartner School of Pharmaceutical Sciences University of Geneva, University of Lausanne Life Sciences Mass Spectrometry

Quai Ernest-Ansermet, 30

$\mathrm{CH}-1211$ Geneva 4

Tel.: +4122 3796344

Fax: +4122 3793332

E-mail: Gerard.Hopfgartner@unige.ch the drug time-course in plasma, since metabolites may be reactive or accumulated and can cause undesired toxicity. ${ }^{[2]}$ In addition, the study of concomitant molecules that can give information regarding the drug mechanism of action or anticipate/detect any undesirable effect has also become of great importance. For this purpose, a new analytical field has emerged this last decade known as metabolomics. ${ }^{[3,4]}$ While NMR is a technique widely used in metabolomics due to its unchallenged identification capabilities, LC-MS has emerged as an attractive technique due to its sensitivity and high throughput when analyzing complex matrices such as plasma or urine.

In order to combine most efficiently both qualitative determination of unexpected drug metabolites or potential biomarkers and quantitative measurements of a parent drug with its metabolites, innovative strategies using LC-MS instrumentation have been investigated since the last decade. Continuous MS instrumentation improvements mainly in terms of resolving power, duty cycle and sensitivity, have allowed the integration of both qualitative and quantitative information (QUAL/ QUAN) acquisition within a single analysis. ${ }^{[5-8]}$ Critical parameters to reach simultaneous QUAL/QUAN capabilities are: i) a short MS(/MS) duty cycle time (ca. 0.1 sec.) for hyphenation with UHPLC-like chromatographic separation; ii) a mass accuracy within 5 ppm for both MS and MS/ MS modes; iii) a resolving power (>30 000) compatible with structural elucidation; and iv) a dynamic range and limits of quantification similar to triple quadrupole instruments. ${ }^{[9]}$ High-resolution mass spectrometers such as Fourier transform-MS (e.g. Orbitrap $\left.{ }^{[10-12]}\right)$ or Quadrupole-TimeOf-Flight (QqTOF) ${ }^{[13,14]}$ provide accurate mass measurements that have become absolutely critical to identify unknown metabolites and/or biomarkers. Furthermore, QqTOF platforms can cope with the duty cycle requirements of QUAL/QUAN experiments.

We present herein a QUAL/QUAN workflow based on a UHPLC-QqTOF platform that combines, within a single 
LC run, an untargeted qualitative approach that aims at discovering unanticipated drug metabolites and also potential biomarkers, together with the quantitative analysis of acetaminophen (APAP) and two of its metabolites, i.e. acetaminophen-glucuronide (APAP-GLU) and acetaminophencysteine (APAP-CYS). The unsupervised qualitative approach consists of an MS ${ }^{\mathrm{ALL}}$ strategy where two TOF MS experiments are successively performed either at low or high collision energy (CE). The low $\mathrm{CE}$ TOF MS experiments are processed by multivariate analysis (MVA), i.e. principal component analysis (PCA), followed by Principal Component Variable Grouping (PCVG) to group the variables according to similarities across all the study samples and to facilitate the determination of APAP and ketorolac related metabolites. Then, structural determination of unanticipated metabolites or biomarker candidates is performed based on the accurate mass of the precursor ion together with the MS/MS spectrum resulting from the corresponding high CE TOF MS experiment. Finally, the last looped experiment is a targeted Product Ion Scan used to quantify APAP and its metabolites by High-Resolution Selected Reaction Monitoring (HR-SRM) mode. This QUAL/QUAN workflow was applied on samples from a population of patients treated successively and randomly with APAP, with a combination of APAP and ketorolac or with a placebo.

\section{Experimental}

\section{Reagents and Materials}

4-(Acetamido)-phenyl- $\beta$-D-glucuronide (APAP-GLU) monosodium salt, $\mathrm{S}$-[5(acetamido)-2-hydroxyphenyl]-cysteine (APAP-CYS) trifluoroacetic salt were obtained from Toronto Research Chemicals (Toronto, Canada). N-(2-hydroxyphenyl)acetamide (APAP) was obtained from Sigma (Buchs, Switzerland). Its deuterated analogue, N-(4-hydroxyphenyl-2,3,5,6d4)-acetamide (APAP-d4) was purchased from Toronto Research Chemicals. S-phenyl-L-cysteine (PHE-CYS) was obtained from Acros Organics (Acros Organics N.V., Geel, Belgium).

Ethanol (EtOH) and methanol $(\mathrm{MeOH})$ solvents were obtained from VWR International (France). Milli-Q water A10 instrument (Millipore, Bedford, MA, USA) was used as deionized water supply. Ammonium acetate and acetic acid were purchased from Fluka (Buchs, Switzerland).

\section{Stock and Plasma Standard Solutions}

Stock solutions of the three analytes were prepared independently in water.
Spiked solutions of human plasma were prepared by adding $10 \mu \mathrm{L}$ of each standard in $970 \mu \mathrm{L}$ of blank plasma. Eight different concentration levels were prepared and aliquoted in $50 \mu \mathrm{L}$ calibration standards and quality control (QC) samples. Concentration of the three analytes ranged from $50 \mathrm{ng} / \mathrm{mL}$ to $10,000 \mathrm{ng} / \mathrm{mL}$. Finally, a 'biological QC' sample was prepared by pooling $10 \mu \mathrm{L}$ of each patient sample (i.e. study samples), mixing thoroughly and aliquoted in $50 \mu \mathrm{L}$ samples before any further sample preparation. This biological QC sample is used to assess data quality throughout the whole batch before processing the study samples.

\section{Study Samples}

A double-blind, randomized, crossover, placebo-controlled study was performed on 11 healthy human volunteers. ${ }^{[15]}$ Drug solutions were prepared for intravenous injections as a $100 \mathrm{~mL}$ solution plus a $0.66 \mathrm{~mL}$ solution in syringe. $1 \mathrm{~g}$ of APAP (Perfalgan ${ }^{\circledR}$, Bristol-Myers Squibb, Baar, Switzerland) or placebo (sodium chloride $0.9 \%$ ) was prepared in a $100 \mathrm{~mL}$ solution and was injected as a $10 \mathrm{~min}$. iv infusion. $20 \mathrm{mg}$ of ketorolac (Tora-Dol ${ }^{\circledR}$, Bristol-Myers Squibb, Baar, Switzerland) or placebo (sodium chloride $0.9 \%$ ) was prepared in $0.66 \mathrm{~mL}$ solution and injected by iv bolus. Blood samples were drawn from the patients after $1 \mathrm{~h}$ and added to ethylenediaminetetraacetic acid tubes (Becton-Dickinson, Franklin Lakes, NJ, USA). Tubes were then centrifuged for 15 min at $900 \mathrm{~g}$ to obtain plasma and stored at $-20^{\circ} \mathrm{C}$ until analysis.

\section{Sample Preparation}

Prior to sample preparation, internal standards were added to a $50 \mu \mathrm{L}$ sample aliquot (i.e. $10 \mu \mathrm{L}$ of PHE-CYS (500 ng/ $\mathrm{mL})$ and $10 \mu \mathrm{L}$ of APAP-d4 (125 ng/mL)). Then, a 4:1 ratio of ice-cold mixture of $\mathrm{MeOH} / \mathrm{EtOH}(1: 1 ; \mathrm{v} / \mathrm{v})$ was added for protein precipitation. Homogenization of the samples was carried out in a thermomixer (Vaudaux-Eppendorf, Buchs, Switzerland) for $10 \mathrm{~min}$ at $15^{\circ} \mathrm{C}$ and 1,400 $\mathrm{rpm}$. Centrifugation was then performed at $4^{\circ} \mathrm{C}$ for $12 \mathrm{~min}$. at $14,000 \mathrm{x} \mathrm{g}$. Supernatant was transferred into new tubes and evaporated to dryness before the reconstitution step in $200 \mu \mathrm{L}$ of ammonium acetate buffer $(\mathrm{pH} 4.75)$ mixed further for $10 \mathrm{~min}$ at $15{ }^{\circ} \mathrm{C}$ and $1,400 \mathrm{rpm}$ (Thermomixer). Finally, $5 \mu \mathrm{L}$ was injected into the LC-MS/ MS system.

\section{UHPLC Conditions}

UHPLC Focused ${ }^{+}$UltiMate 3000 RSLC (Dionex, Germering, Germany) was used for UHPLC separation and was configured in a binary high-pressure gra- dient mode. Mobile phase A was $5 \mathrm{mM}$ ammonium acetate buffer ( $\mathrm{pH}$ 4.75) and mobile phase $\mathrm{B}$ was $\mathrm{MeOH}$. The starting composition of mobile phase was set at $5 \% \mathrm{~B}$ and held for $0.5 \mathrm{~min}$. It was then increased linearly up to $25 \% \mathrm{~B}$ over 3.5 min and held for $0.8 \mathrm{~min}$. and finally up to $90 \% \mathrm{~B}$ in $2.2 \mathrm{~min}$. and held for $2 \mathrm{~min}$. The column was then re-equilibrated at $5 \%$ $\mathrm{B}$ for $8.9 \mathrm{~min}$. and the total run time was $18 \mathrm{~min}$. The sub- $2 \mu \mathrm{m}$ column was a YMC Ultra-HT Hydrosphere C18, 2.0 mm i.d. $\times$ 150 mm (YMC Europe GmBH, Dinslaken, Germany) with KrudKatcher UHPLC filter $0.1 \mathrm{~mm}$ i.d. $\times 0.5 \mu \mathrm{m}$ (Phenomenex, Torrance, CA). Constant LC flowrate was set at $350 \mu \mathrm{L} / \mathrm{min}$. and column oven was kept at a temperature of $40{ }^{\circ} \mathrm{C}$.

\section{Mass Spectrometry}

Experiments were performed on a TripleTOF 5600 (AB Sciex, Concord, Canada) equipped with a DuoSpray ionization source used in APCI mode for the calibrant channel and in electrospray mode for the LC eluent. The positive ion spray floating voltage was set at $5.5 \mathrm{kV}$. The automated calibration device system (CDS) was run every three samples to perform automatically the TOF calibration. The source temperature was set at $425^{\circ} \mathrm{C}$ and the curtain gas and the auxiliary gases GS1 and GS2 were set at 25, 50 and 70 (laboratory frame), respectively. The TripleTOF $5600 \mathrm{MS}$ was operated at a resolving power of about 30,000 in TOF MS mode and at a resolving power of 20,000 in MS/MS high-sensitivity mode.

For the LC-MS acquisition four consecutive periods of different duration were devised. Each of these periods runs the three looped MS(/MS) experiments as follows: the first one is a TOF MS experiment at low collision energy $(\mathrm{CE}=10 \mathrm{eV})$ monitoring from $\mathrm{m} / \mathrm{z} 50$ to 1000 with an accumulation time of $75 \mathrm{~ms}$. The second one is the same TOF MS experiment but carried out at a higher collision energy (CE $=50 \mathrm{eV}$ ). For both experiments, declustering potential (DP) is set at $50 \mathrm{~V}$. The third looped experiment differs for each period and consists in the product ion scan (PIS) of APAP-GLU $(m / z$ 328.1) for the first period (from 0 to $4.2 \mathrm{~min}$.). For the second period (4.2 to $4.7 \mathrm{~min}$.), APAP-CYS $(\mathrm{m} / \mathrm{z} 271.1)$ is selected as precursor ion. For the third period (4.7 to $5.5 \mathrm{~min}$.), APAP $(\mathrm{m} / \mathrm{z} 152.5)$ and APAP-d4 $(\mathrm{m} / \mathrm{z}$ 156.5) are fragmented using the quadrupole resolution set to open to allow both precursor ions to be selected. For the last period (5.5 to $11 \mathrm{~min}$.), ISTD PHE-CYS $(\mathrm{m} / \mathrm{z}, 198.1)$ is targeted for fragmentation. The optimized parameters for these different MS(/MS) experiments are reported in Table 1. 
Table 1. MS parameters of the third Product lon Scan experiment used for the quantification of APAP and two of its metabolites with their respective ISTD

\section{APAP (\& APAP-d4)}

Q1 $[m / z]$

Q1 resolution

152.5

Open

$\mathrm{CE}[\mathrm{eV}]^{\mathrm{a}}$

$\mathrm{DP}[\mathrm{V}]^{\mathrm{a}}$

Accu. time [ms]

50

Scan range $[\mathrm{m} / \mathrm{z}]$

$100-200$
APAP-CYS

271.1
Unit
31
55
50

$100-200$
APAP-GLU

328.1

Unit

18.5

103

50

$100-700$

\section{PHE-CYS}

198.1

Unit

25

10

50

100-200

${ }^{\mathrm{a}} \mathrm{CE}=$ Collision Energy, $\mathrm{DP}=$ Declustering Potential

\section{Analytical Sequence}

The analytical sequence began with the injection of human blank plasma (plasma 00) and human blank plasma spiked only with the ISTD (plasma 0). Then, a series of calibration standard and QC samples were injected for the quantitative aspect of the assay. Finally, the biological QC sample was injected at the beginning $(n=6)$ and at the end $(n=6)$ of the study samples as well as every six study samples $(n=1)$. The injection order of study samples was randomized using random function from Excel 2010 paired with samples number. After the last biological QC sample, a series of QC samples were analyzed. Blank samples (buffer) were run at the beginning of the sequence, after plasma 0 and in between calibrations and QC series.

\section{Data Processing}

Analytes quantification was performed using the MultiQuant 2.1 software (AB Sciex) using the MQ4 integration algorithm and a Gaussian smooth width of 2.0 points.

The metabolomics processing workflow was done with MarkerView 1.2.1 software (AB Sciex) as follows: peak picking was performed with a minimal RT of 5 scans, a minimal spectral width of $20 \mathrm{ppm}$ and a subtraction offset of 30 scans. Then samples were aligned with a RT tolerance of $0.1 \mathrm{~min}$ and a $\mathrm{m} / \mathrm{z}$ tolerance of $20 \mathrm{ppm}$. Sample normalization was done by using the two ISTD (i.e. APAP-d4, PHE-CYS), the latter being globally excluded from the principal component analysis (PCA) afterwards. PCA was carried out with a Pareto scaling on features eluting from 0.9 to 10 min and Principal Component Variable Grouping (PCVG) was performed using an angle of $40^{\circ}$ and a number of principal components explaining $90 \%$ of the variance, excluding groups with less than ten features. The group order was assigned according to their magnitude. Elemental formula determination was done by Formula Finder (AB Sciex) and MS $^{\text {ALL }}$ scan processing was performed with a prototype plug-in from the PeakView v1.1 software (AB Sciex).

\section{Results and Discussion}

The knowledge of biotransformation products of drugs and their associated pharmacokinetic profiles is important for the understanding of undesirable effects appearing during a treatment. Quantification of metabolites is especially critical when there is formation of reactive metabolites that can modify cellular and/or protein functions. In the case of acetaminophen (APAP), it is well described that even at therapeutic doses, 5 to $10 \%$ of the parent compound is oxidized by CYP450 into a quinone imine derivative[16] that reacts with intracellular nucleophiles such as glutahione (GSH). This adduct is not directly detectable in plasma but one of its related metabolite, i.e. APAP-CYS, is. ${ }^{[16]}$ Another interesting metabolite to consider is the acetaminophen-glucuronide (APAP-GLU) as it is the most concentrated in plasma and therefore represents a major excretion pathway for APAP. In a QUAL/QUAN workflow based on a high-resolution MS instrument, the quantification of APAP and two of its metabolites is carried out in a slightly different way than on triple quadrupole MS instrumentation commonly used in bioanalysis. Indeed, one takes advantage of the high resolving power of the MS instrument to perform the quantitative assay in HR-SRM mode instead of Q1/Q3 unit resolution SRM.

\section{LC-HR-SRM Quantification of APAP, APAP-GLU and APAP-CYS in Plasma Study Samples}

The LC-HR-SRM assay developed for the quantification of APAP and two of its metabolites was validated and will be described in detail elsewhere (manuscript in preparation). Briefly, HR-SRM chromatograms are built with the third PIS experiments by extracting for each period a fragment ion specific to the selected precursor ion with a narrow $\mathrm{m} / \mathrm{z}$ window of 0.02 $0.04 \mathrm{u}$. Then, each individual HR-SRM chromatogram is processed with APAP-d4 being used as ISTD to quantify APAP and PHE-CYS for the quantification of
APAP-CYS and APAP-GLU. According to standard validation criteria (i.e. accuracy and precision of QC samples within $15 \%$ of the target values or within $20 \%$ at the lower limit of quantification), the HRSRM quantification range of each analyte was linear from 50 to $10^{\prime} 000 \mathrm{ng} / \mathrm{mL}$ starting from a $50 \mu \mathrm{L}$ human plasma aliquot. Fig. 1 shows representative LC-HR-SRM chromatograms of a blank plasma sample, at the lower limit of quantification, and of a study sample from the APAP-ketorolac administration group (i.e. patient 9).

Fig. 2 shows the quantitative results as box-and-whisker plots. For the group of patients treated only with APAP, average concentrations $(\mathrm{n}=11)$ were $6,650 \mathrm{ng} / \mathrm{mL}$ for APAP, $154.2 \mathrm{ng} / \mathrm{mL}$ for APAP-CYS and $8,750 \mathrm{ng} / \mathrm{mL}$ for APAP-GLU. For the group treated with a combination of APAP and ketorolac, average concentrations $(\mathrm{n}=$ 11) were $6,160 \mathrm{ng} / \mathrm{mL}$ for APAP, $140.6 \mathrm{ng} /$ $\mathrm{mL}$ for APAP-CYS and $8,430 \mathrm{ng} / \mathrm{mL}$ for APAP-GLU.

Interestingly, differences between the two groups were observed to be less than $10 \%$ indicating an independence of the APAP metabolism from the ketorolac metabolism. The presence of ketorolac metabolites circulating in plasma has been shown to be limited to a single detected substance,

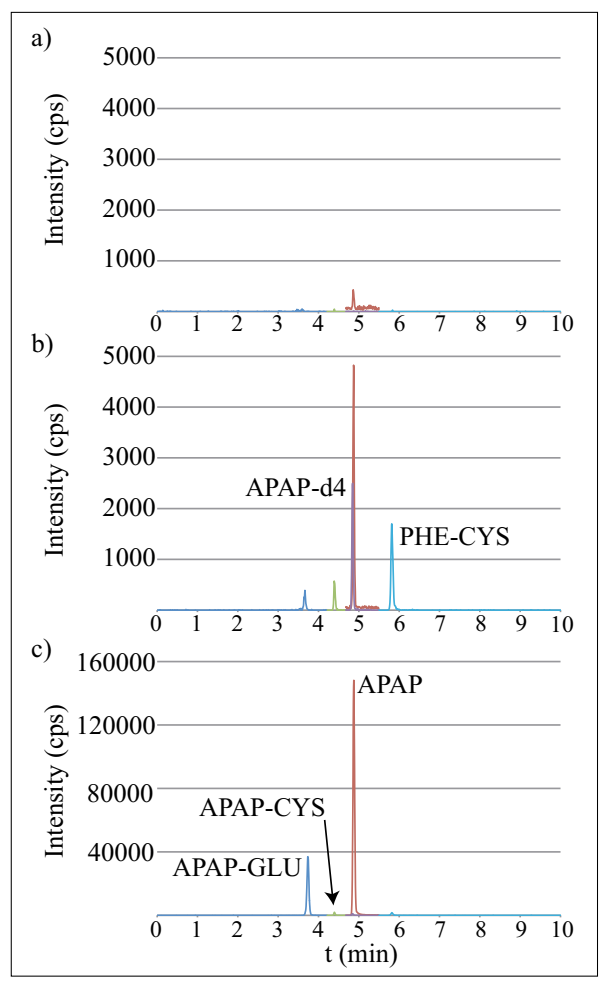

Fig. 1. HR-SRM traces of APAP $(m / z 152.1 \rightarrow 110.060)$, APAP-GLU $(\mathrm{m} / \mathrm{z} 328.1 \rightarrow 152.071)$ and APAP-CYS $(\mathrm{m} / \mathrm{z} 271.1 \rightarrow 140.016)$ a) for a blank plasma sample (plasma 00), b) at the lower limit of quantification (LLOQ), and c) for the patient 9 with APAP and ketorolac administration (measured concentrations: APAP $=4,316 \mathrm{ng} / \mathrm{mL}$, APAP-GLU $=6,394 \mathrm{ng} / \mathrm{mL}$ and APAP-CYS = $120.4 \mathrm{ng} / \mathrm{mL})$. 


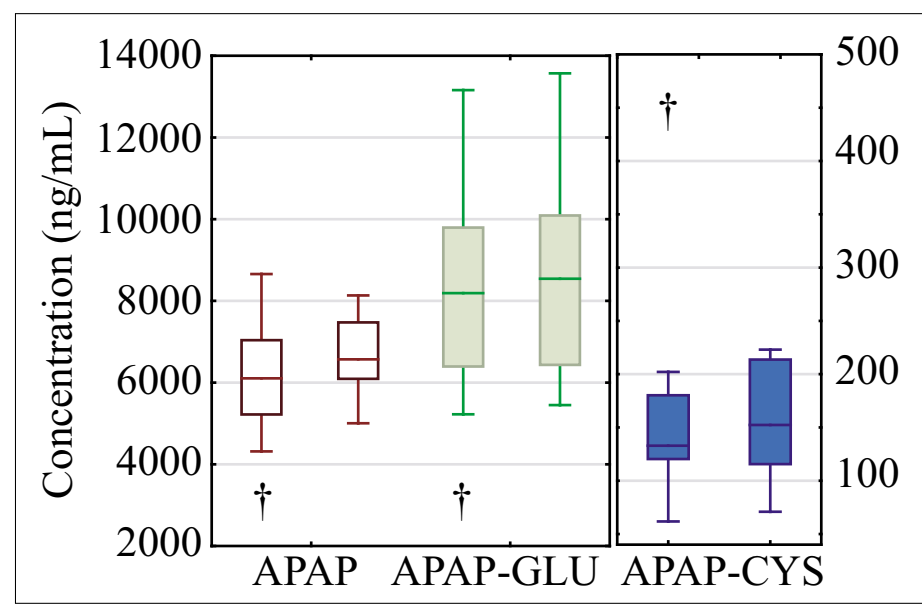

Fig. 2. Box-andwhisker plots for APAP, APAP-GLU and APAP-CYS quantitative results of patients treated with APAP and ketorolac (leftside marked with $\dagger$ ) or treated only with APAP (right-side). a $p$-hydroxyl metabolite, accounting for less than $5 \%$ of the parent drug[17,18] indicating a low metabolism in hepatic cells. In contrast, phase I and phase II metabolism of APAP is extensively hepatic ${ }^{[16]}$ mainly with the formation of a glucuronide and a sulfated conjugated products. In consequence, metabolism pathways do not seem to be influenced by each other in case of co-administration as performed in this study. Finally, for the third group of samples, none of these three analytes were detected.

\section{Exploratory Qualitative Analysis of APAP and Ketorolac Metabolites}

\section{Considerations about the Analytical Sequence Design for a QUAL/QUAN Assay}

A QUAL/QUAN assay requires a dedicated LC-MS(/MS) method with different types of unsupervised and supervised MS(/ MS) experiments, but also a specific analytical sequence design. Indeed, the analytical sequence contains calibration standards and quality control samples for the quantitative analysis of the study samples, but it should also contain several replicates of a biological QC sample to assess data quality throughout all the batch for performing the qualitative data processing of the study samples. ${ }^{[19]}$ As commonly accepted in bioanalysis the plasma standard samples, used to build the calibration curve to quantify APAP, APAP-GLU and APAPCYS, were run first followed by series of validation QC samples bracketing the study samples. For the qualitative aspect of the assay, the biological QC, consisting of the pool of all study samples aliquots, was also bracketing the study samples by several replicates, as well as injected every six study samples. The presence of the biological QC within the sequence is critical for several reasons: i) it is used to ensure the analytical level of performance throughout the sequence; ii) it helps to determine the different parameters of the peak picking process (i.e. $\mathrm{m} / \mathrm{z}$ and retention time toler- ances); iii) the replicates bracketing the study samples are useful to eliminate peaks detected that have a significant drift during the sequence. Moreover, randomization of study samples is absolutely critical to ensure reliable and high quality of data. ${ }^{[19]}$

\section{Metabolomics Dataset Processing to Find Potential Candidates}

The qualitative aspect of this study was to investigate unanticipated metabolites related to APAP and ketorolac. The comprehensive metabolomics dataset processing workflow has already been described elsewhere. ${ }^{[19]}$ Briefly, the low CE TOF MS experiment was used to extract features consisting of $<m / z$, RT $>$ pairs by taking advantage of the high mass accuracy $(<5 \mathrm{ppm})$ and resolving power $(30,000)$ of the MS instrument. The peak picking process was optimized using the biological QC runs to adjust parameters related to peak finding, alignment and filtering of these $<\mathrm{m} / \mathrm{z}$, $\mathrm{RT}>$ features. Then, a principal component analysis (PCA) with Pareto scaling was performed. The first five principal components (PC) represented $70.4 \%$ of the overall variance observed in the dataset. Fig. 3 a shows the scores plot for PC1 and PC2

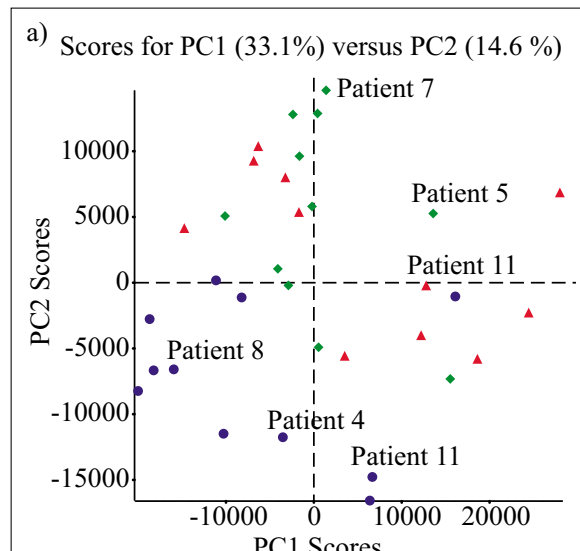

Fig. 3. a) Scores plot for PC1/PC2 obtained for the PCA analysis with Pareto scaling (patients group legend: disk = Placebo, triangle $=$ APAP, diamond $=$ APAP + ketorolac), and $b$ ) Loadings plot for PC1/PC2 after group assignment with PCVG $\left(90 \%\right.$ variation and angle of $40^{\circ}$, excluding groups with less than 10 features). b) Loadings for PC1 (33.1\%) versus PC2 (14.6\%)

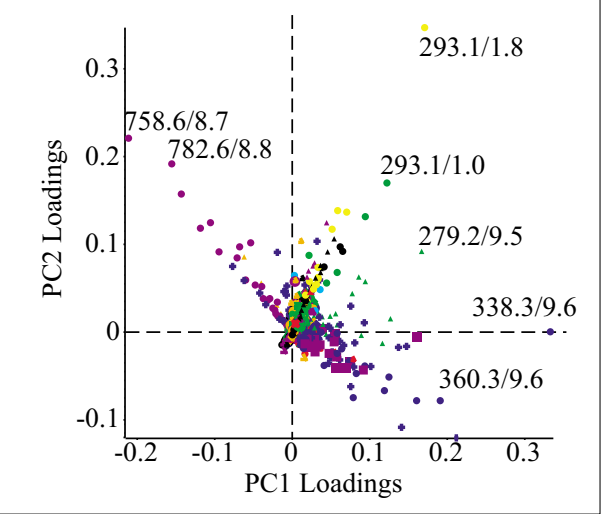

where samples from the group of patients treated with a placebo are separated from the samples of the two other groups.

Principal Component Variable Grouping (PCVG) was then used to group variables according to the similarity of their profiles across the samples. ${ }^{[20]}$ Fig. $3 b$ shows the PCA loadings plot for PC1 and PC2 with the resulting 43 groups found by PCVG.

As one of the goals is to detect metabolites related to APAP and ketorolac, the PCVG groups containing APAP, APAPGLU and APAP-CYS were evaluated first. As a matter of fact the group 13 gathers all three molecules, and contains 90 features in total that are present in samples from the APAP group of patients, as well as from the APAP + ketorolac group, but not from the placebo group. After removing isotopes, adducts (e.g. Na-, $\mathrm{K}-, \mathrm{NH}_{4}^{-}$ adducts), dimers or noise, a short list of 27 candidates of interest remained including the three known analytes (i.e. APAP, APAP-GLU and APAP-CYS) (Fig. 4).

\section{Structural Identification of the Potential Candidates}

The workflow for the structural and molecular elucidation of these candidates is illustrated hereafter using the feature $<m / z$ 232.0, RT=5.7 min. $>$ as an example. First the accurate mass has to be retrieved for the elemental composition determination. An averaged $\mathrm{m} / \mathrm{z}$, value of 232.0280 $\pm 0.0001(n=22)$ was obtained for all the samples from the group of patients treated with APAP or treated with APAP and ketorolac. This $m / z$ value was submitted to the mass calculator of PeakView software with the following limitations: a maximum composition of $\mathrm{C}_{100} \mathrm{H}_{500} \mathrm{~N}_{20} \mathrm{O}_{20} \mathrm{P}_{10} \mathrm{~S}_{10}$, an $\mathrm{m} / \mathrm{z}$ error less than $5 \mathrm{ppm}$ and a ring plus double bonds (RDB) equivalent ranging from -0.5 to 50 . A total of 10 elemental compositions was proposed. Therefore, a 


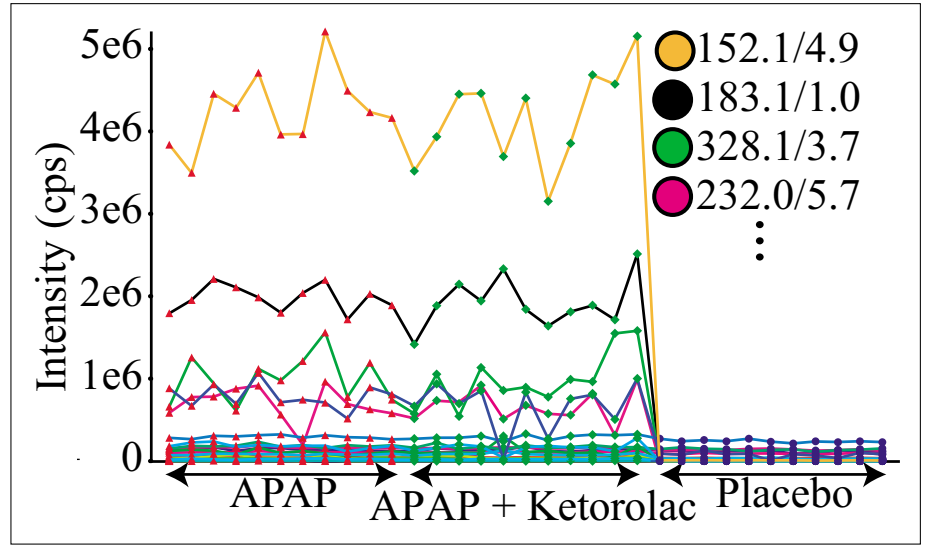

representative MS spectrum (i.e. patient 2 from the APAP + ketorolac group) showing the most intense signal for the $\mathrm{m} / \mathrm{z}$ value of 232.0280 was selected in order to use the Formula Finder software. Indeed, this algorithm compares also the isotopic distribution in addition to the exact mass when proposing elemental formula. Consequently, by applying the same limitations with an additional $10 \%$ tolerance on the intensity of the isotopic pattern, the list could be narrowed down to two candidates, i.e. $\mathrm{C}_{8} \mathrm{H}_{10} \mathrm{NO}_{5} \mathrm{~S}$ and $\mathrm{C}_{10} \mathrm{H}_{7} \mathrm{~N}_{3} \mathrm{O}_{2} \mathrm{P}$. At this stage there is a high probability that the first hit corresponds to the APAP-sulfated metabolite when comparing to the APAP chemical formula (i.e. $\mathrm{C}_{8} \mathrm{H}_{9} \mathrm{NO}_{2}$ ). But to confirm this assumption, the next step was to use the high CE TOF MS spectrum for a structural confirmation by MS/MS. Compared to the data-dependent MS/MS spectra acquisition (i.e. IDA-mode), the $\mathrm{MS}^{\mathrm{ALL}}$ strategy does not require any precursor ion selection in Q1 and thus no collision energy tuning. Indeed, all the precursor ions eluting at a certain retention time are fragmented altogether in the high $\mathrm{CE}$ TOF MS experiment at a generic CE value. The main advantage of this technique is the unsupervised and fast acquisition mode of MS/MS spectra, however with more unrelated fragment ions due to the lack of selectivity in the precursor ion selection. In addition, the assignment of each fragment ion to its respective precursor ion is challenging in terms of data processing. In Fig. 5, the low and high CE TOF MS spectra of the candidate eluting at $5.7 \mathrm{~min}$. are shown after data deconvolution. By assigning the main fragment ions, the candidate was proposed as the sulfated metabolite of APAP. Following the same data processing workflow for the other candidates, the elucidation of a mercapturate metabolite of APAP and of a circulating ketorolac with a high degree of confidence was also possible. For the candidates with ambiguous identification a fraction collection approach as shown by Staack et al. ${ }^{[21]}$ might be needed.

\section{Conclusion}

A fast duty cycle, high resolving power UHPLC-QqTOF platform has been described for performing QUAL/ QUAN experiments that allow the simultaneous quantification of APAP and two of its metabolites (APAP-glucuronide and APAP-cysteine), as well as qualitative metabolomic investigations in a set of human plasma study samples. Formal identification and characterization of APAP-sulfate and APAP-mercapturate metabolites was performed using a metabolomics data processing based on PCA and PCVG, as well

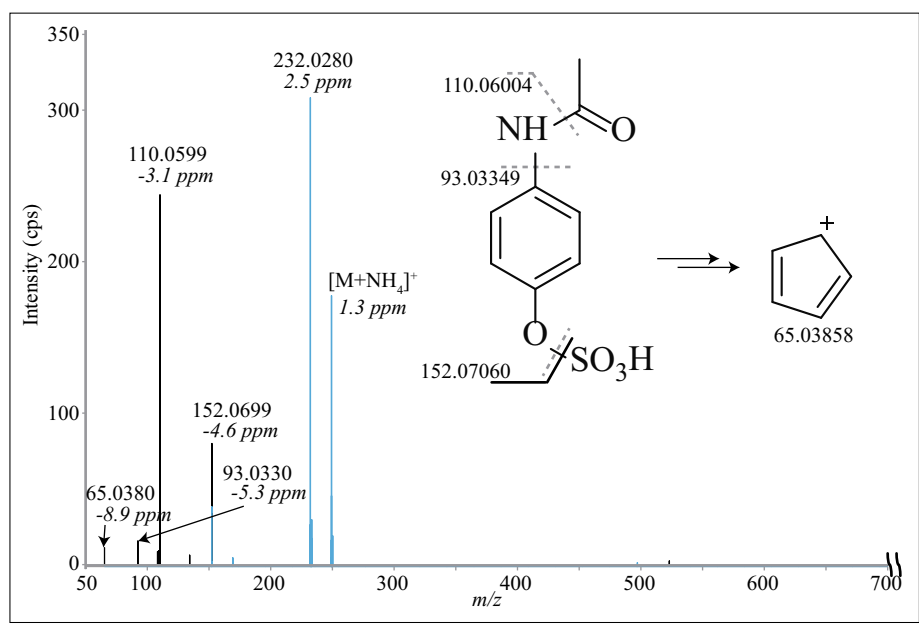

Fig. 4. Profile plot across samples of PCVG group 13 corAPAP-related metabolites. responding to the

as on the MS ${ }^{\mathrm{ALL}}$ data acquisition strategy. Further data processing can be performed using the same data set to identify potential biomarkers from other PCVG groups.

\section{Acknowledgements}

The authors would like to acknowledge Youssef Daali (University Hospital of Geneva, Switzerland) for providing the study samples that were used for this work and also for providing blank plasma used for the quantification method. They also would like to acknowledge AB Sciex (Concord, Canada) and Dionex (Germering, Germany) for fruitful collaborations.

Received: January 13, 2012

[1] G. Evans, in 'A handbook of bioanalysis and drug metabolism', Ed. G. Evans, CRC Press, 2004.

[2] D. A. Smith, H. van de Waterbeemd, D. K Walker, in 'Pharmacokinetics and Metabolism in Drug Design', Eds. D. A. Smith, H. van de Waterbeemd, D. K. Walker, R. Mannhold, H Kubinyi, H. Timmerman, Wiley-VCH Verlag $\mathrm{GmbH}, \mathbf{2 0 0 1}$, p. 102

[3] J. K. Nicholson, J. C. Lindon, E. Holmes, Xenobiotica 1999, 29, 1181.

[4] J. K. Nicholson, J. Connelly, J. C. Lindon, E. Holmes, Nat. Rev. Drug Discovery 2002, 1, 153.

[5] G. Hopfgartner, C. Husser, M. Zell, J. Mass Spectrom. 2003, 38, 138.

[6] T. V. Olah, D. A. McLoughlin, J. D. Gilbert, Rapid Commun. Mass Spectrom. 1997, 11, 17.

[7] G. K. Poon, G. Kwei, R. Wang, K. Lyons, Q. Chen, V. Didolkar, C. E. C. A. Hop, Rapid Commun. Mass Spectrom. 1999, 13, 1943.

[8] N. Zhang, S. T. Fountain, H. Bi, D. T. Rossi, Anal. Chem. 2000, 72, 800 .

[9] W. Korfmacher, Bioanalysis 2011, 3, 1169.

[10] K. P. Bateman, M. Kellmann, H. Muenster, R. Papp, L. Taylor, J. Am. Soc. Mass Spectrom. 2009, 20, 1441

[11] A. Koulman, G. Woffendin, V. K. Narayana, H. Welchman, C. Crone, D. A. Volmer, Rapid Commun. Mass Spectrom. 2009, 23, 1411.

[12] N. R. Zhang, S. Yu, P. Tiller, S. Yeh, E. Mahan, W. B. Emary, Rapid Commun. Mass Spectrom. 2009, 23, 1085 .

[13] M. Wrona, T. Mauriala, K. P. Bateman, R. J. Mortishire-Smith, D. O'Connor, Rapid Commun. Mass Spectrom. 2005, 19, 2597.

[14] Y. Q. Xia, J. Lau, T. Olah, M. Jemal, Rapid Commun. Mass Spectrom. 2011, 25, 2863.

[15] K. Ing Lorenzini, M. Besson, Y. Daali, D Salomon, P. Dayer, J. Desmeules, Basic Clin. Pharmacol. Toxicol. 2011, 109, 357.

[16] J. G. M. Bessems, N. P. E. Vermeulen, Crit. Rev. Toxicol. 2001, 31, 55.

Fig. 5. Low (light trace) and high CE (dark trace) TOF MS spectra used for structural elucidation of APAP-SULF metabolite with the MS ${ }^{\text {ALL }}$ strategy.
[17] E. J. Mroszczak, F. W. Lee, D. Combs, F. H. Sarnquist, B. L. Huang, A. T. Wu, L. G. Tokes, M. L. Maddox, D. K. Cho, Drug Metab. Dispos. 1987, 15.

[18] A. T. Wu, I. J. Massey, J. Chromatogr., B: Biomed. Sci. Appl. 1990, 534.

[19] R. Mohamed, E. Varesio, G. Ivosev, L. Burton, R. Bonner, G. Hopfgartner, Anal. Chem. 2009, $81,7677$.

[20] G. Ivosev, L. Burton, R. Bonner, Anal. Chem. 2008, 80, 4933 .

[21] R. F. Staack, E. Varesio, G. Hopfgartner, Rapid Commun. Mass Spectrom. 2005, 19, 618. 